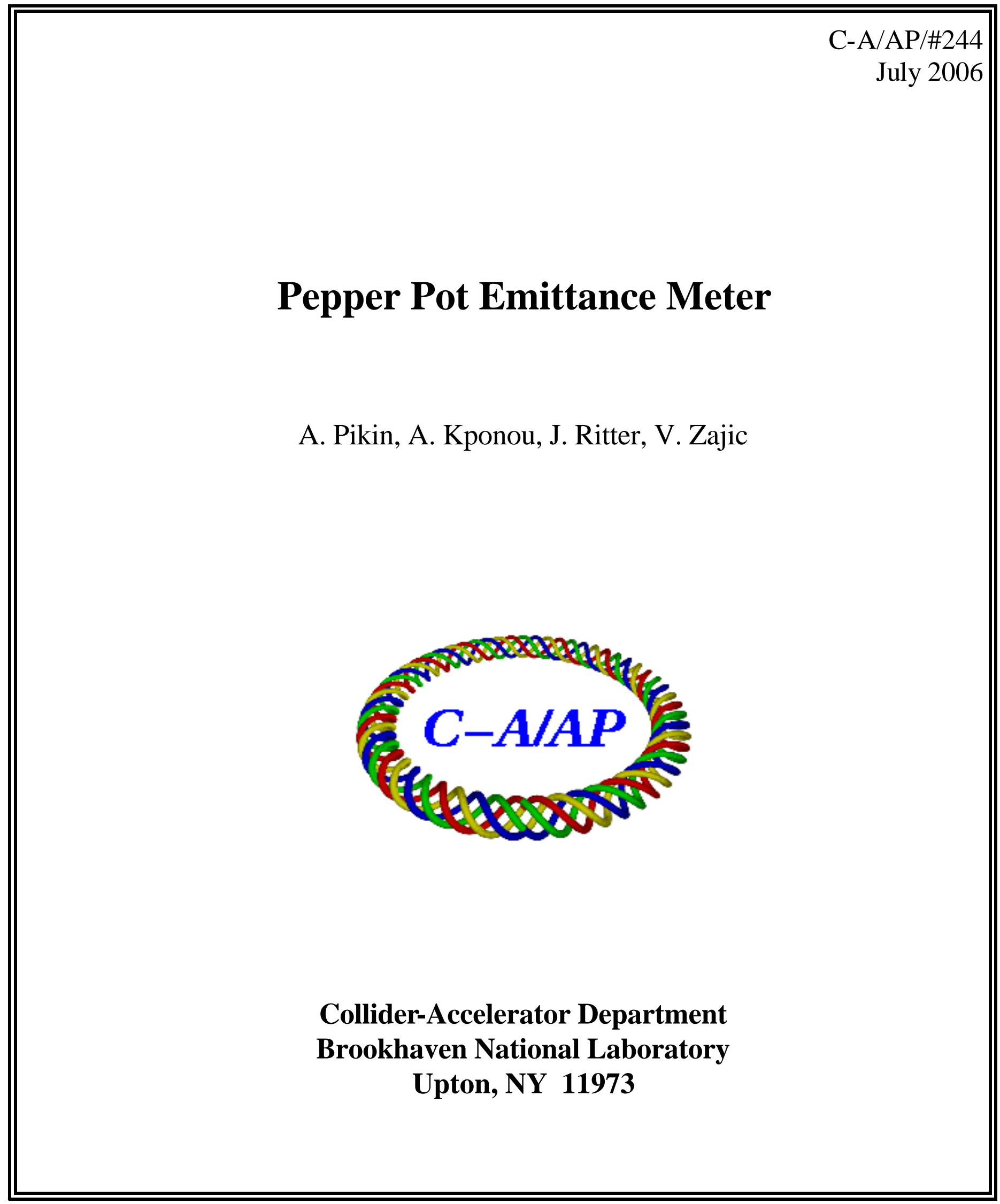




\title{
Pepper pot emittance meter
}

\author{
A. Pikin, A. Kponou, J. Ritter, V. Zajic
}

BNL C-AD Technote

\section{Introduction}

To measure the emittance of the ion beam primarily extracted from EBIS Test Stand, a new emittance meter has been designed and built. The motivation to use pepper pot emittance meter (PPEM) is a need to measure both X- and Y-components of the beam emittance simultaneously in one shot and to present all emittance- and profile-related data in real time. The device is similar to GSI design [1]. A goal was to make a universal device, which can be used in several positions on a beam line for a range of ion energies from several keV/nucleon to several hundred $\mathrm{keV} /$ nucleon.

\section{Design and operation of the pepper pot emittance meter.}

The main components of the pepperpot are the mask of pinholes, the microchannel plate (MCP) and phosphor screen assembly, the surface silvered mirror, and the CCD camera with an attached macro zoom lens.

The beam mask is made of a tungsten foil $100 \mu \mathrm{m}$ thick. The pinholes with diameter $100 \mu \mathrm{m}$ are spaced $2 \mathrm{~mm}$ horizontally and vertically, covering a sensitive area with diameter of $43 \mathrm{~mm}$. To prevent thermal deformation of the mask, the tungsten foil is sandwiched between two $0.5 \mathrm{~mm}$ thick copper plates. These supporting plates have the same structure of holes as the tungsten mask but the holes in these plates are larger (diameter $0.5 \mathrm{~mm}$ ) to prevent shielding of the mask. The mask assembly is insulated from the ground to allow for ion beam current measurement, with an applied bias voltage to suppress secondary electrons. The central pinhole of the mask is closed for beamlet identification purpose.

The MCP is a chevron type, with a gain of up to $10^{7}$. It is backed by a P46 phosphor screen, several millimeters away. The distance between the $\mathrm{MCP} /$ phosphor assembly and the mask is adjustable from 5 to $50 \mathrm{~mm}$, to provide optimization for non-overlapping beamlet images with maximum diameter.

A mirror, which is mounted behind the phosphor screen, reflects the image of beamlets through the vacuum window to the camera. The macro zoom lens allows the camera to be mounted close to the window, thus making the instrument compact.

To be able to measure time-resolved emittances of the extracted ion beam, the acquisition or "shutter" time of the CCD camera and decay time of the phosphor screen should be much shorter than the ion pulse length. The monochrome video camera, Sony XCD-SX910 with IEEE 1394 digital interface, has a shortest "shutter" time of $10 \mu \mathrm{s}$. The phosphor used for our screen has decay time $300 \mathrm{~ns}$ (90\% to $10 \%$ of intensity).

An isometric view of the device is presented in Fig. 1. The whole PPEM with camera is mounted on a single 8 " standard ConFlat flange. 
MCP is shielded from secondary electrons and stray light with a copper foil. A photograph of PPEM without shield is presented in Fig. 2.

In operation, the camera is set to capture a single image, or a sequence of images, and save them to files, using the software provided by the manufacturer. Locally developed software, described in the following section, analyzes the files. Under development is software to automate capture and processing in a single step.

\section{Data processing}

The analysis of captured images followed these steps:

1. Loading. A grayscale bitmap image from CCD camera is loaded and displayed, either in its actual size, or scaled to fit the computer display. Subsequent processing of the image is possible only if it has 8-bit amplitude resolution (256 channels).

2. Background determination. The image consists of beamlets and Poisson distributed noise. The median $(\mathrm{M})$ of this distribution is not affected by the contribution from the beam. The mean and median of the noise (background) are very close to each other and have amplitude between 10 and 20 for the existing amplitude resolution. The median is found after using the Shell-sort algorithm (for about $10^{\wedge} 6$ pixels) to sort the pixel intensities [2].

3. Noise filtering. Intensity of each pixel is replaced by the median - not average - of its intensity and those of the surrounding 8 pixels. The program has the option to repeat this step. The effect of noise filtering was found to be equivalent to averaging a sequence of about 10 images (Figure 3).

4. Background subtraction. a Noise factor (F) (about 1.3 to 1.5 with noise filtering, and 2.2 to 3.0 without it) is chosen, based on our experience. Pixel intensities, which have amplitude less than or equal to $\mathrm{M} * \mathrm{~F}$ are set to zero, while intensities greater than $\mathrm{M} * \mathrm{~F}$ are reduced by subtracting $\mathrm{M}$. The quality of the noise subtraction can be checked visually by changing the black color in the bitmap color table to yellow. Pixel intensities of any residual noise - dark shades of gray - become clearly visible, if the noise factor is too low and/or if the noise filter has not been applied.

5. Projections and gridlines. Making a one-to-one association between beamlet images and holes in the mask is essential for the method to work. This consists of the finding the boundaries (demarcated by gridlines) of the peaks in both the horizontal and vertical projections in such a way that aberration effects are treated correctly. Some of the cells bounded by the horizontal and vertical gridlines contain the beamlets of interest to us. Small manual adjustments of the gridlines can be made by dragging them with the mouse if the aberrations are not correctly handled by the program (Figure $4 \mathrm{a}$ and $4 \mathrm{~b}$ ).

6. Identifying blocked hole. The association between beamlets and holes is straightforward if one hole in the mask - the central one in our case - is closed. For each cell, the difference between the weighted average of the 8 adjacent cells (with weight 1 
for the 4 adjacent side cells and weight 0.5 for the 4 adjacent diagonal cells) and the cell's total intensity is calculated. The cell, for which the difference is a maximum, should correspond to the missing beamlet of the blocked hole. The empty cell in the beam image with the grid is marked. If the missing beamlet is mis-identified, it can be repositioned with a mouse click, before the next step in the analysis. See Figure 5.

7. Gaussian fitting. The vertical and horizontal projections are sums of the contributions from the columns and rows of the image. If the contributions from adjacent columns or rows overlap, they can be separated by fitting multiple gaussians to the projections by the least square method. The centroid, mean square root deviation, and total intensity of each peak in Figure $2 \mathrm{a}$ and $2 \mathrm{~b}$ are good approximations to start the fitting procedure. The gaussian fit usually yields emittances within $2 \%$ of the emittances calculated without the fit, but it smudges any beam aberrations.

8. Emittance calculation. Knowing the relative positions of the "origins" of the mask and phosphor screen, and camera's resolution in pixels per phosphor screen distance, the horizontal and vertical displacements of all beamlet's pixels from the corresponding mask hole (from step 6, above) can be readily calculated. Knowing the mask-to- microchannel plate detector distance, $\mathrm{x}-\mathrm{x}^{\prime}$ and $\mathrm{y}-\mathrm{y}^{\prime}$ data for horizontal and vertical phase plots are obtained, from which the rms emittance is calculated. Since we are measuring emittance at the mask, the $\mathrm{x}$ and $\mathrm{y}$ values in the plot are those of the associated holes. The vertical and horizontal projections are used to calculate the $x-x^{\prime}$ and $y-y^{\prime}$ emittances. A useful feature of the program is that it allows the user to examine the contributions to the emittance of beam falling on individual rows and columns of holes in the mask.

9. Recalculation. The emittance is automatically recalculated after any gridline shifting or blocked hole repositioning. It can be recalculated manually after additional noise filter passes, or changing the value of the noise factor $F$ used in the background subtraction.

10. Presentation. All data and images can be printed and copied, via the clipboard, to other applications such as word processors (MS Word). An example of the program presentation for a sample ion beam is presented in Figure 6.

11. Saving. When a sequence of images is taken and averaged, the resulting image can be saved as a device independent bitmap file, so that the averaging does not have to be repeated.

The program was written in $\mathrm{C}++$, using MS Visual $\mathrm{C}++6.0$.

\section{References}

1. Hoffmann, W. Barth, P. Forck, A. Peters, P. Strehl, D. A. Liakin, Proc. 9th BIW 2000, Cambridge, USA, pp. 432-439.

2. Robert Sedgewick, Analysis of Shellsort and Related Algorithms, Fourth Annual European Symposium on Algorithms, Barcelona, September, 1996. 


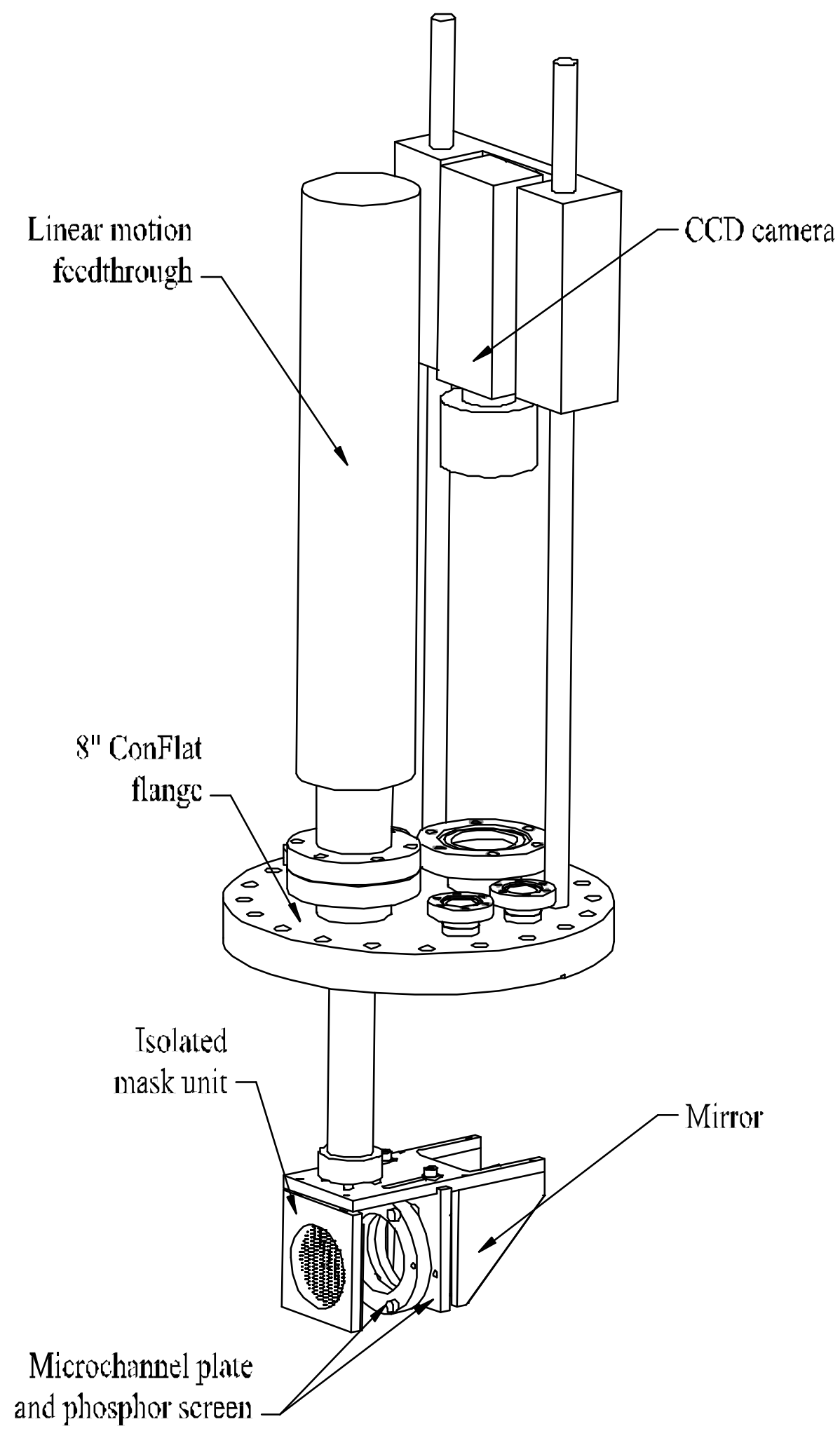

Fig. 1. Design of a pepper pot emittance meter. 


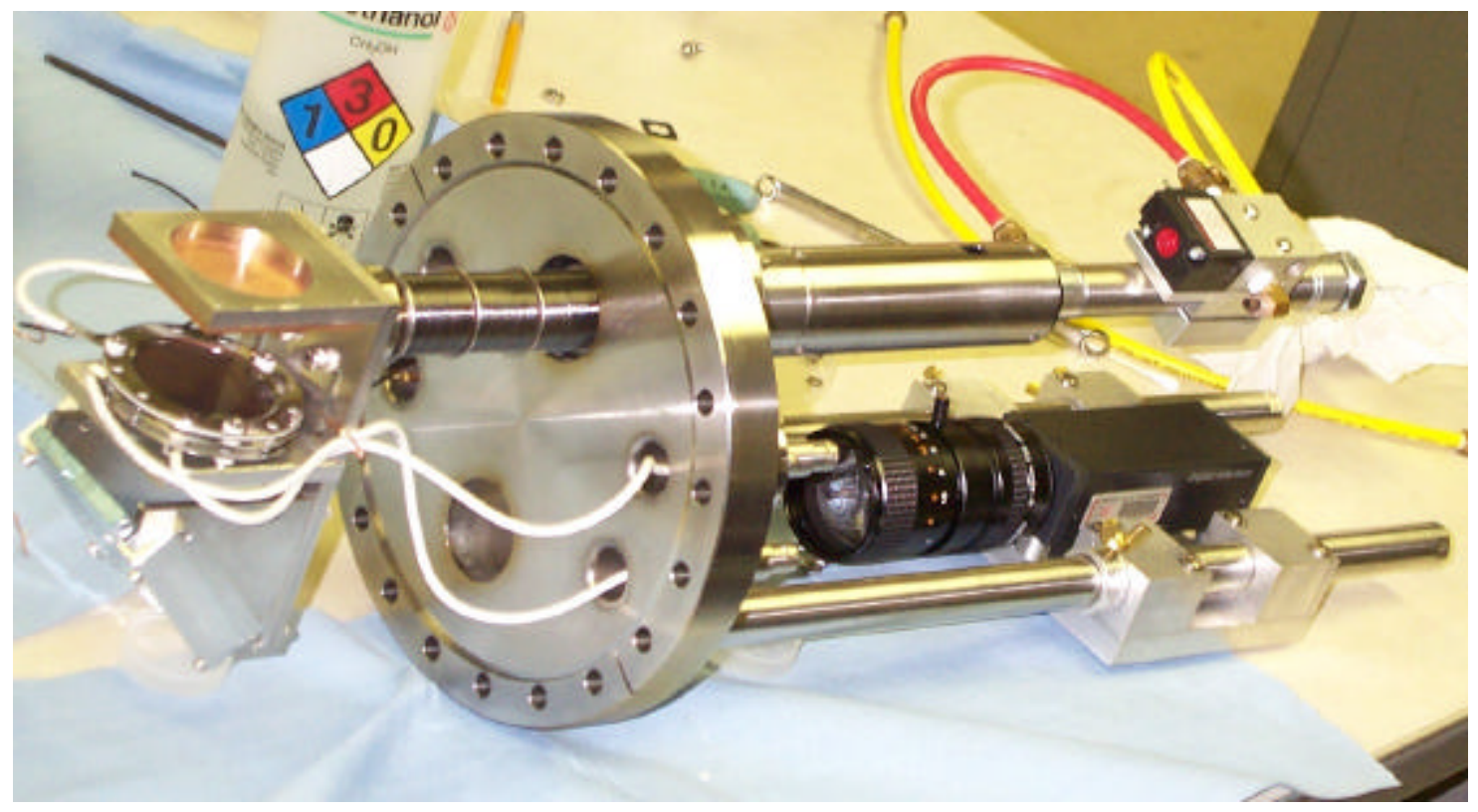

Fig. 2. A photograph of the pepper pot emittance meter with head retracted (no shield).

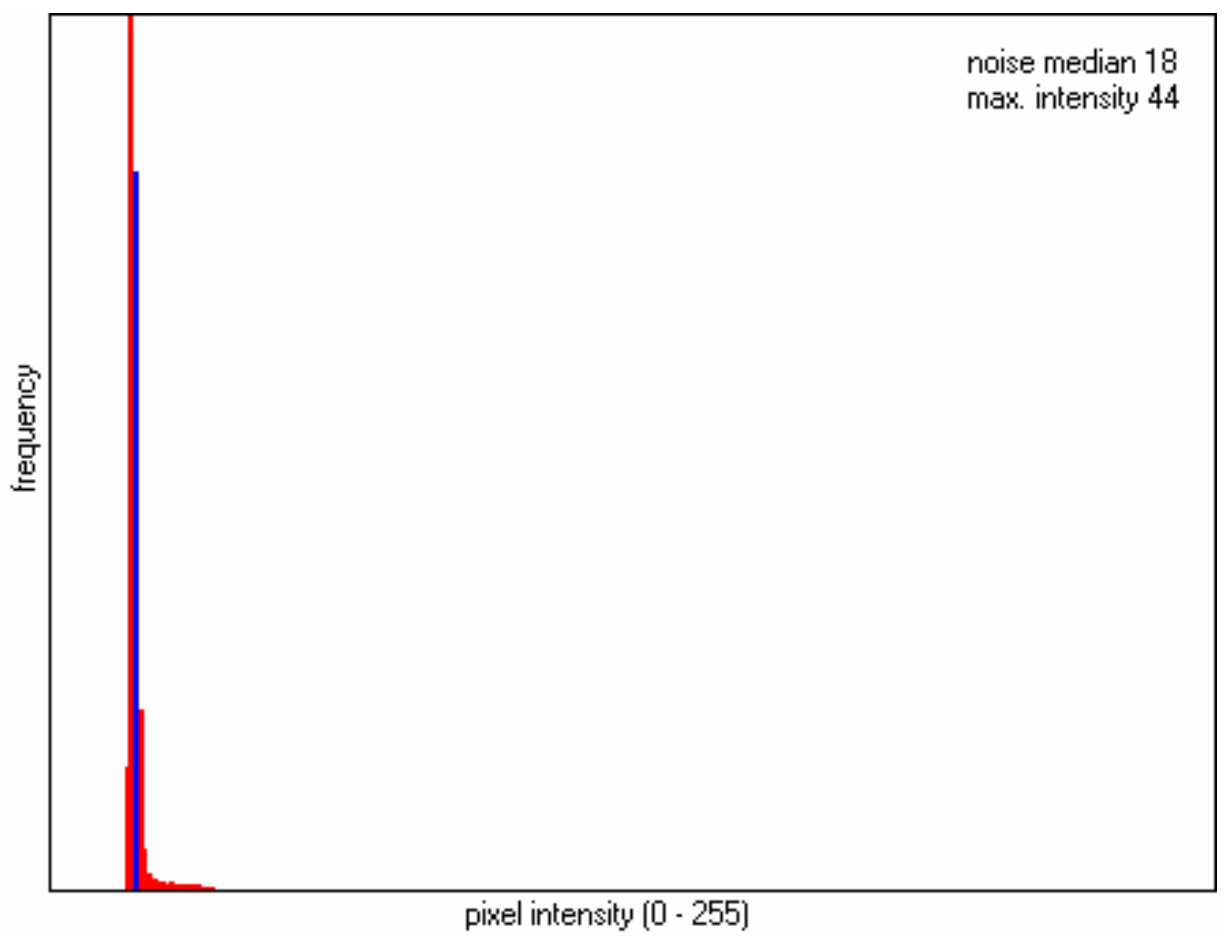

Figure 3. Pixel intensity distribution. 


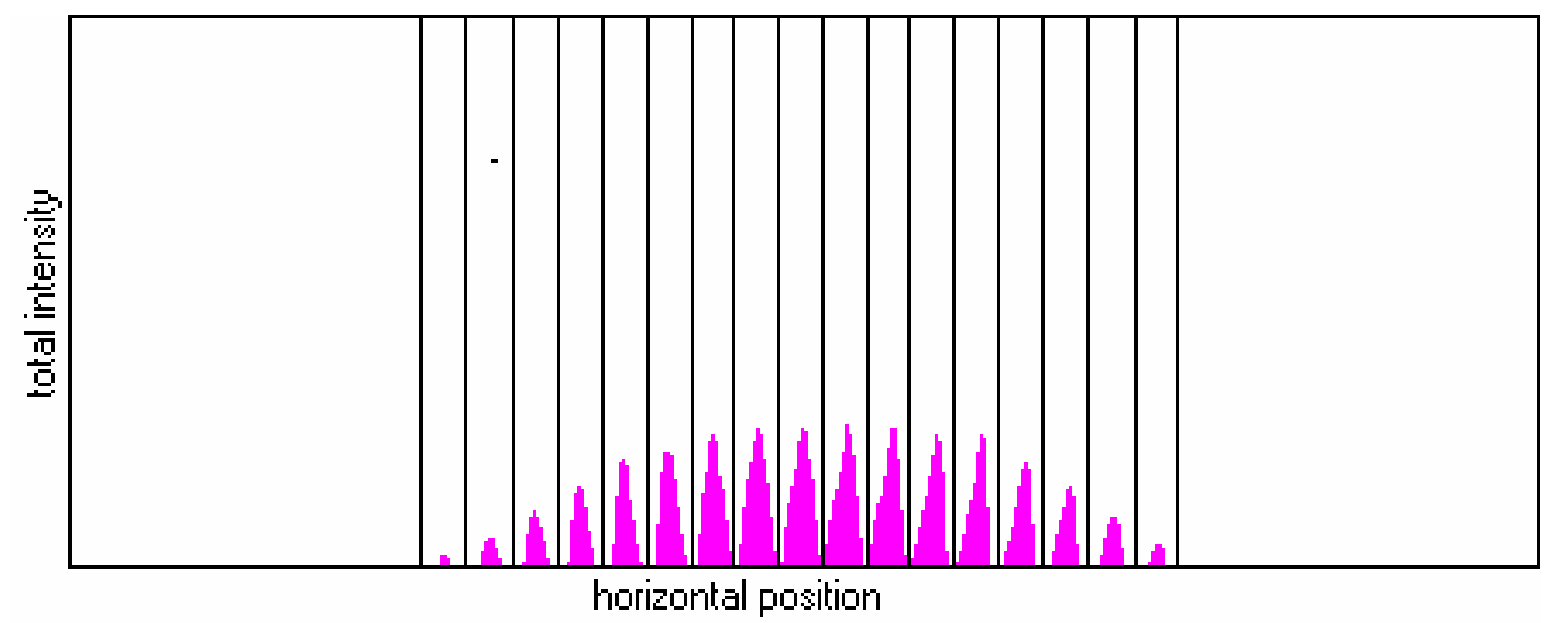

Figure 4a. Horizontal projection

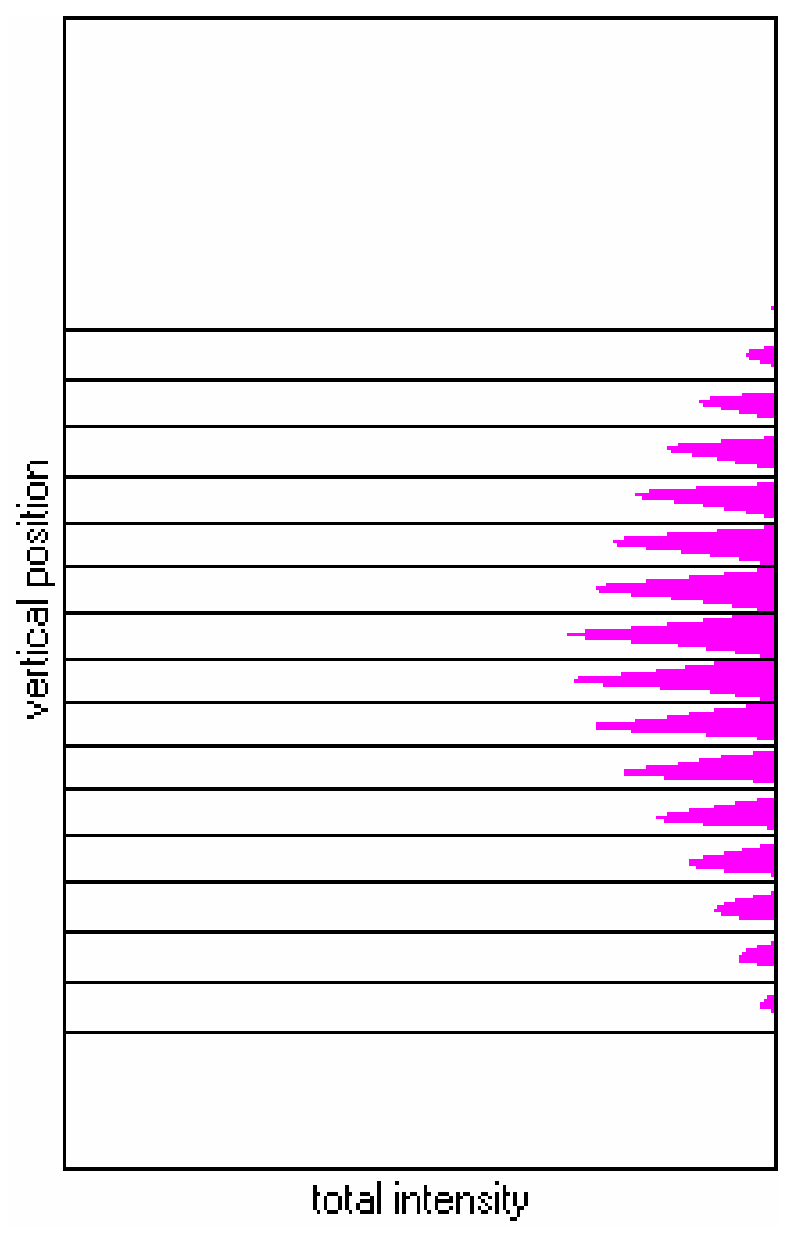

Figure 4b.Vertical projection. 


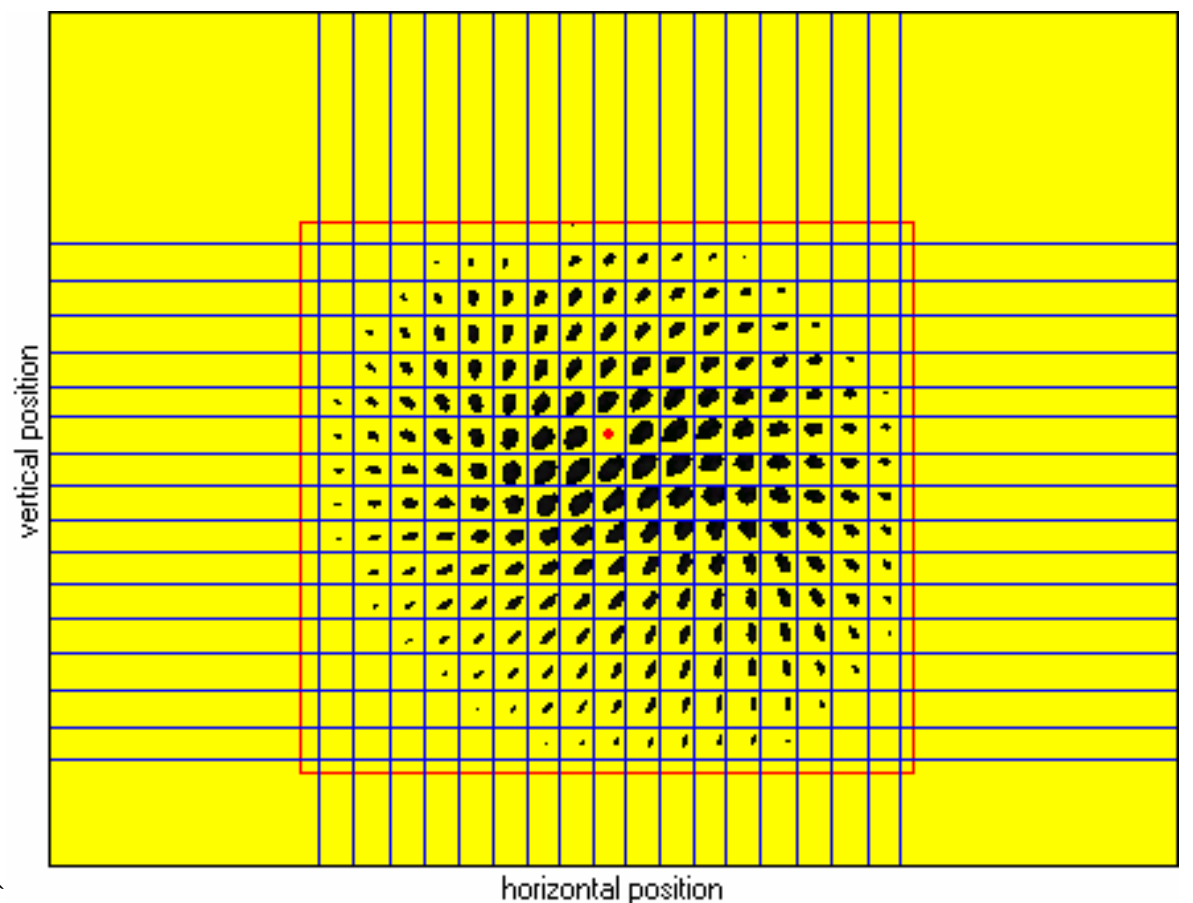

Figure 5. Gridlines and missing beamlet superimposed on a beam image, black (zero intensity) replaced by yellow.
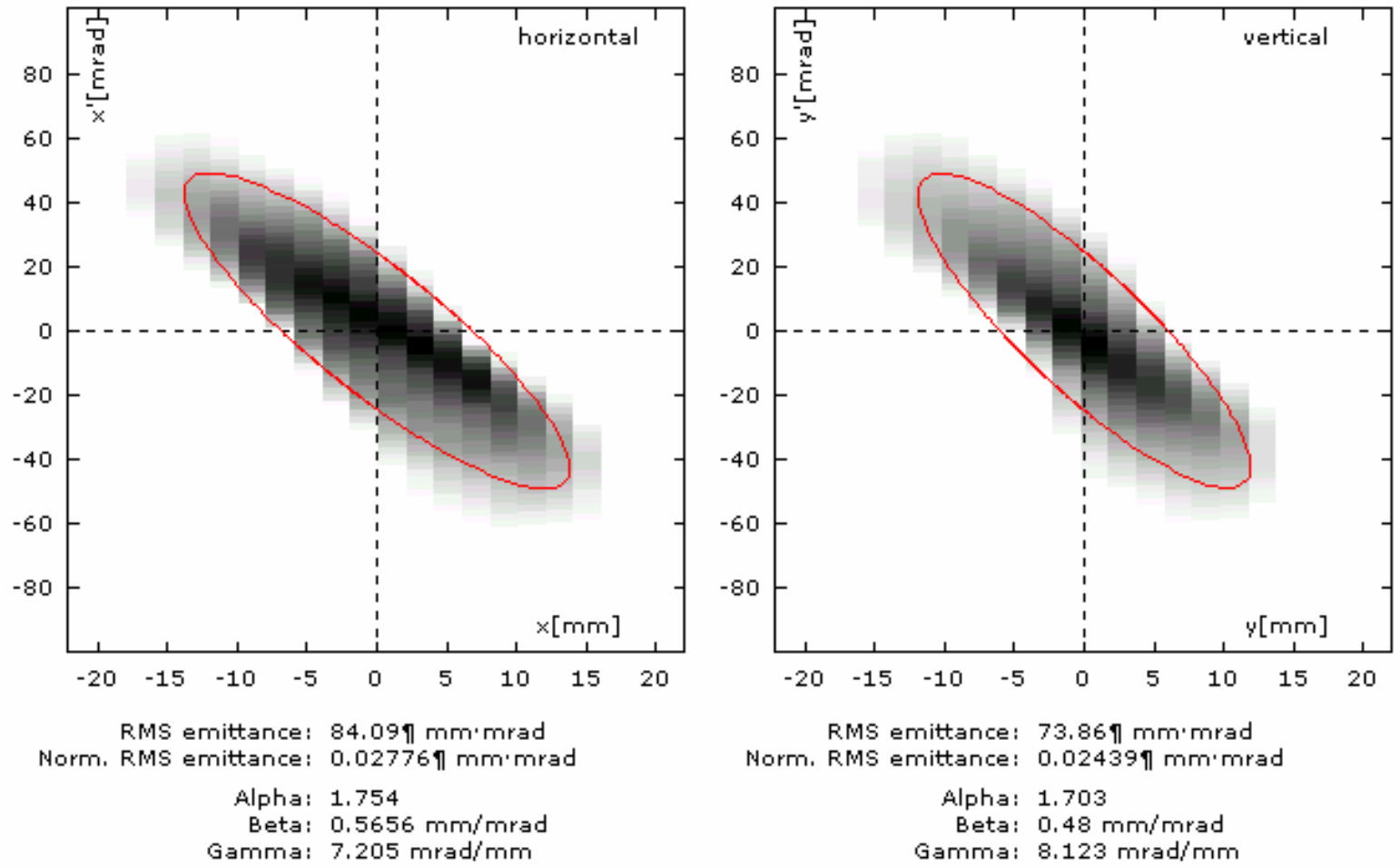

Figure 6. Phase plots and results of the rms emittance calculation. The $4 *$ rms emittance ellipses are plotted. 